\title{
Noninvasive assessment of cerebral artery stenoses from anatomic computed tomography angiography
}

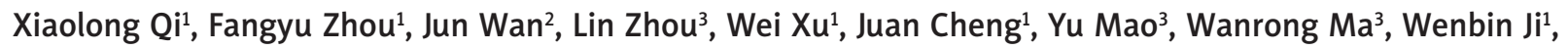 \\ Changqing Yang ${ }^{1}$
}

${ }^{1}$ Institute of Digestive Disease, Tongji Hospital, Tongji University School of Medicine, Shanghai, China

2Division of Interventional Radiology, Shanghai Jing'an District Central Hospital, Shanghai, China

${ }^{3}$ Division of Cardiology, Tongji Hospital, Tongji University School of Medicine, Shanghai, China

Postep Kardiol Inter 2014; 10, 1 (35): 18-20

DOI: $10.5114 /$ pwki.2014.41461

\begin{abstract}
A bstract
Trans-stenotic pressure gradient (TSPG) and fractional flow reserve (FFR) are well-validated hemodynamic indices to assess the ischemic severity of arterial stenoses. However, they have significant restrictions in practice due to invasiveness and high cost. Recently, a noninvasive assessment of FFR from computed tomography (CT) angiography has been well validated in evaluating functional coronary stenoses. We present a case of a 65 -year-old woman with cerebral artery stenoses demonstrated by clinical symptoms, transcranial Doppler ultrasound and CT angiography, aiming to transplant the noninvasive functional diagnosis, for the first time, to cerebral artery stenoses and test a computational workflow of noninvasive TSPG and FFR.
\end{abstract}

Key words: cerebral artery stenoses, trans-stenotic pressure gradient, fractional flow reserve, noninvasive.

\section{Introduction}

Trans-stenotic pressure gradient (TSPG) and fractional flow reserve (FFR) are two well-validated functional indices to assess the ischemic severity of arterial stenoses [1-3]. They have been demonstrated as a promising tool to identify patients who would likely benefit from revascularization [4-6]. However, TSPG and FFR measurement has significant restrictions in clinical practice due to its invasiveness and high cost.

A novel noninvasive assessment of FFR from anatomic computed tomography (CT) angiography has been well validated in evaluating functional coronary stenoses [1-3].

The study aims to transplant the functional diagnosis, for the first time, to cerebral artery stenoses and develop a noninvasive assessment of cerebral TSPG (TSPG $\left.{ }_{n i}\right)$ and FFR $\left(\right.$ FFR $\left._{\mathrm{ni}}\right)$.

\section{Case report}

A 65-year-old woman who had symptoms of cerebral ischemia was recruited and gave written informed consent. Approval was obtained from the ethical committees of Tongji Hospital, Tongji University. Transcranial Doppler ultrasound and CT angiography were performed according to a recognized protocol by two technicians who had at least 5 years' vascular examination experience.

For the $\mathrm{TSPG}_{n i}$ and $\mathrm{FFR}_{\mathrm{ni}}$ interpretation, all CT images were imported into Medical Imaging Control Software MIMICS10.0 where the posterior cerebral artery (PCA) was reconstructed. Three dimensional virtual arteries were then exported from MIMICS10.0 as a virtual reality modeling file to the developed workflow. The inlets and outlets were defined according to the position from which the physiological data were recorded. The closed surface was "meshed" into approximately 1.2 million internal tetrahedra, in preparation for the computational fluid dynamics analysis. Measured flow data and universal central venous pressure were imported, processed, and applied to the inlet and outlet as boundary conditions, and a definition file was created that fully specified the model for the computational fluid dynamics solver ANSYS13.0. This solved the unsteady momentum Navier-Stokes and continuity equations with finite element analysis. TSPG ${ }_{n i}$ and $F F R_{n i}$ were then calculated. An opti-

\section{Corresponding author:}

Prof. Changqing Yang, Tongji Hospital, Tongji University School of Medicine, 389 Xin Cun Road, 200065 Shanghai, China, phone: +86 21 66111076, e-mail: cqyang@tongji.edu.cn, changqingyang@126.com

Received: 18.08.2013, accepted: 6.12.2013. 


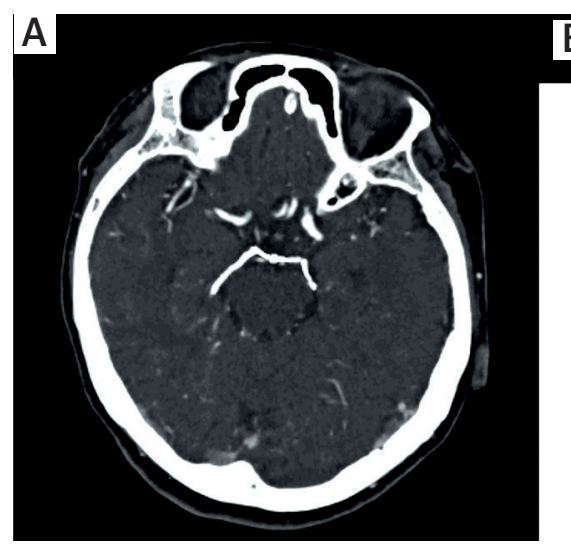

B

C
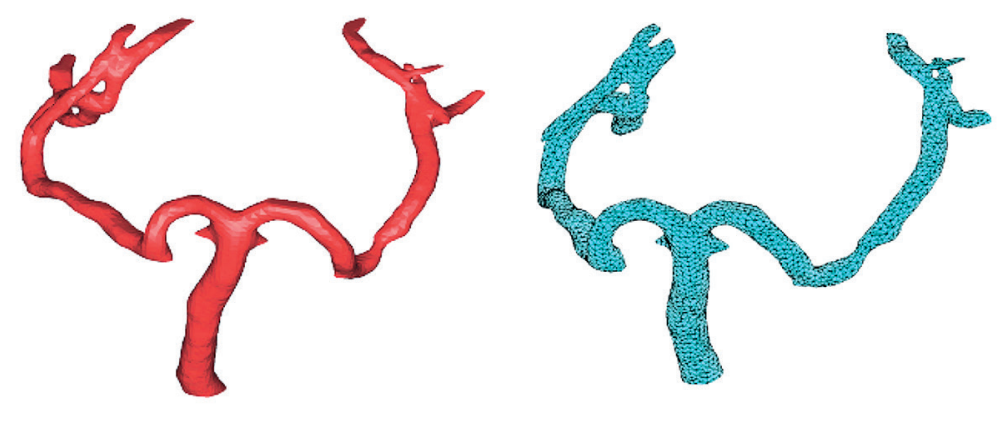

Figure 1. Three-dimensional modeling and finite element analysis of posterior cerebral artery. A - Two-dimensional model reconstruction of the transverse section through cerebral CT angiography. B - Three-dimensional geometric model was extracted from surrounding tissues. C - Geometric model was meshed with local refined tetrahedral finite element

\section{A}



$F F R_{n}$ 0
B
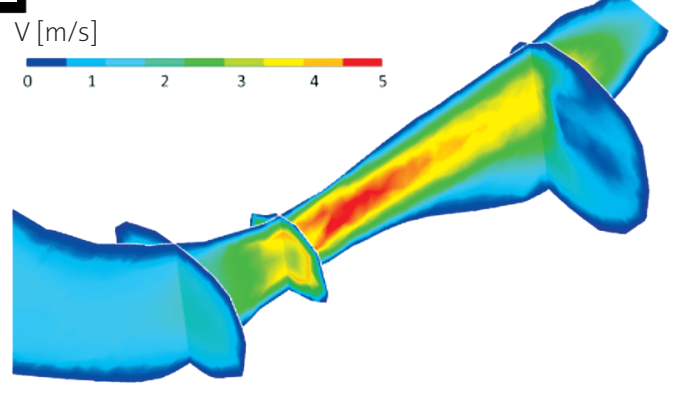

C

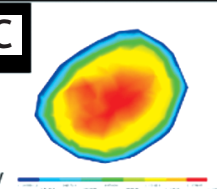



Figure 2. Anatomical obstruction of posterior cerebral artery with/without hemodynamic stenoses. A - CT angiography suggested mild stenoses (15\%, red arrow) on the right side of the PCA and moderate stenoses (65\%, blue arrow) on the left side. Cerebral TSPG ${ }_{n i}$ and FFR $_{n i}$ of the right PCA were $31.6 \mathrm{~mm} \mathrm{Hg}$ and 0.43 ; they were $59.2 \mathrm{~mm} \mathrm{Hg}$ and 0.22 for the left side. B - The velocity distribution in coronal section was computed. C - There are three transverse sections around the stenoses. The increase and offset of velocity were well simulated

mization process was used to determine the microvascular resistance for the patient and we averaged the resistance to produce a generic value applicable to the whole cohort. This approach demonstrated the most effectively predictive capability of the model with minimal personalized data.

Two-dimensional models of the PCA in transverse planes were reconstructed (Figure $1 \mathrm{~A}$ ). 3D geometric models of the PCA were separated (Figure 1 B). Finite element computational fluid dynamics of ANSYS11.0 was applied to mesh the models. Thereafter, a well-defined mathematical model was obtained (Figure $1 \mathrm{C}$ ).
Cerebral TSPG ${ }_{n i}$ and FFR $_{n \text { in }}$ were successfully computed. The peak systolic velocity (PSV) of the PCA was $117 \mathrm{~cm} / \mathrm{s}$ (left) and $92 \mathrm{~cm} / \mathrm{s}$ (right). Computed tomography angiography images suggested that the level of stenoses in the PCA was $60 \%$ (left) and 15\% (right). According to novel assessment, TSPG ${ }_{n i}$ and FFR $_{n i}$ of the right side of the PCA were $31.6 \mathrm{~mm} \mathrm{Hg}$ and 0.43 . For the left side, they were $59.2 \mathrm{~mm} \mathrm{Hg}$ and 0.22 (Figure $2 \mathrm{~A}$ ). The velocity distribution in the coronal plane indicated that the velocity remained equal at the proximal and distal stenoses. However, it increased significantly from $1.5 \mathrm{~m} / \mathrm{s}$ to $5.0 \mathrm{~m} / \mathrm{s}$ at the site of stenoses (Figure $2 \mathrm{~B}$ ). In addition, it showed 
that the rate of blood flow increased obviously and central speed shifted to the narrow (Figure $2 \mathrm{C}$ ).

\section{Discussion}

Digital subtraction angiography is still treated as a "gold standard" to evaluate "significant" cerebral artery stenoses. However, drawbacks, such as invasiveness and lack of lesion-specific functional diagnosis, greatly limit its clinical worth [7-9]. A large number of clinical trials have proved the great value of TSPG and FFR, which are well-defined functional indices to evaluate the ischemic significance of artery stenoses [4-6]. Recently, the results of a series of international multicenter clinical trials suggested the highly diagnostic performance of a novel noninvasive FFR assessment from anatomic CT angiography (FFR $\left.{ }_{C T}\right)$ [1-3]. In the study concerning 159 vessels in 103 patients, the diagnostic accuracy, sensitivity and specificity of FFR $_{\text {CT }}$ were $84.3 \%, 87.9 \%$ and $82.2 \%$, respectively. Area under the receiver-operator characteristics curve (AUC) was 0.90 [2]. From the results of another study carried out in 252 stable patients from 17 centers in 5 countries, the diagnostic accuracy, sensitivity and specificity of FFR $\mathrm{CT}_{\text {T }}$ plus CT were 73\%, 90\% and 54\%. FFR was associated with improved discrimination (AUC: $0.81 ; 95 \% \mathrm{Cl}: 0.75-0.86 ; p<0.001$ ) [1].

Our previous studies have successfully demonstrated a novel method computed from CT angiography to functionally evaluate the severity of vascular disease [3]. The study aims at investigating, for the first time, a noninvasive approach to evaluate functional cerebral artery stenoses by TSPG ${ }_{\text {ni }}$ and $\mathrm{FFR}_{\text {ni }}$. The results for the left side of the PCA were $65 \%$ stenoses, PSV $=117 \mathrm{~cm} / \mathrm{s}, T_{S P G}$ $=59.2 \mathrm{~mm} \mathrm{Hg}, \mathrm{FFR}_{\mathrm{ni}}=0.22$, which suggested functional and anatomic moderate stenoses. For the right side, they were $15 \%$ stenoses, PSV $=92 \mathrm{~cm} / \mathrm{s}$, TSPG ${ }_{n i}=31.6 \mathrm{~mm} \mathrm{Hg}$ and $\mathrm{FFR}_{\mathrm{ni}}=0.43$, respectively. From the results, the diagnostic performance of cerebral TSPG $\mathrm{ni}_{\mathrm{i}}$ and $\mathrm{FFR}_{\mathrm{ni}}$ was generally consistent with the diagnosis of transcranial Doppler ultrasound and CT angiography.

\section{Conclusions}

This is the first study to introduce a novel noninvasive assessment of cerebral TSPGni and FFRni to evaluate "significant" cerebral artery stenoses and their hemodynamics. It is therefore believed that the novel method might be potentially useful in evaluating the functional severity of cerebral ischemia and making clinical decisions of revascularization. Although the diagnostic performance is limited due to the small sample and lack of in vivo values of TSPG and FFR, the results are encouraging and the model will now be optimized with greater patient numbers and invasive values analysis.

\section{Acknowledgments}

Xiaolong Qi, Fangyu Zhou and Jun Wan - equal contributor.

\section{References}

1. Min JK, Leipsic J, Pencina MJ, et al. Diagnostic accuracy of fractional flow reserve from anatomic CT angiography. JAMA 2012; 308: 1237-1245.

2. Koo BK, Erglis A, Doh JH, et al. Diagnosis of ischemia-causing coronary stenoses by noninvasive fractional flow reserve computed from coronary computed tomographic angiograms. Results from the prospective multicenter DISCOVER-FLOW (Diagnosis of Ischemia-Causing Stenoses Obtained Via Noninvasive Fractional Flow Reserve) study. J Am Coll Cardiol 2011; 58: 1989-1997.

3. Qi X, Lv H, Zhou F, et al. A novel noninvasive method for measuring fractional flow reserve through three-dimensional modeling. Arch Med Sci 2013; 9: 581-583.

4. De Bruyne B, Manoharan G, Pijls NH, et al. Assessment of renal artery stenoses severity by pressure gradient measurements. J Am Coll Cardiol 2006; 48: 1851-1855.

5. Bley TA, Johnson KM, François CJ, et al. Noninvasive assessment of transstenotic pressure gradients in porcine renal artery stenoses by using vastly undersampled phase-contrast MR angiography. Radiology 2011; 261: 266-273.

6. Wasilewski J, Mirota K, Hawranek M, et al. Invasive and non-invasive fractional flow reserve index in validation of hemodynamic severity of intracoronary lesions. Postep Kardiol Inter 2013; 9: 160-169.

7. Lloyd-Jones D, Adams R, Carnethon M, et al. Heart disease and stroke statistics - 2009 update: a report from the American Heart Association Statistics Committee and Stroke Statistics Subcommittee. Circulation 2009; 119: e21-e181.

8. Howell GM, Makaroun MS, Chaer RA. Current management of extracranial carotid occlusive disease. J Am Coll Surg 2009; 208: 442-453.

9. Kobayashi A. Endovascular approaches for acute ischaemic stroke: the current evidence and organizational issues. Postep Kardiol Inter 2012; 8: 216-219. 